\title{
GALILEO Y LA POLÉMICA SOBRE LA EXPERIMENTACIÓN
}

Jaime Andrés Areiza Serna*

\begin{abstract}
A I momento de indagar sobre la experimentación en Galileo Galilei, 4 es difícil identificar qué tipo de experimentos, efectivamente, - realizó. Esto se debe, fundamentalmente a que al abordar la obra de Galileo, no se logra diferenciar qué experimentos realizó y cuáles no. Además, nuestra concepción de la experimentación moderna, enturbia nuestra indagación. Y eso es lo que, de alguna manera, vamos a tratar de responder aquí: ¿Galileo hacía experimentos?
\end{abstract}

Para lograrlo, se partirá de la idea de "experimento" desde la intuición más básica: "[...] como un modo de conocer algo inmediatamente antes de todo juicio formulado sobre lo aprehendido" (Ferrater Mora 1181). Y, adicionalmente, de la distinción entre experimentos míticos, experimentos imaginarios -0 de pensamiento ${ }^{1}$ - y experimentos reales.

\section{Experimentos míticos}

\section{Misa en el Duomo (Catedral de Pisa)}

Cuenta la leyenda que se encontraba el joven Galileo, con aproximadamente 17 años y siendo estudiante de Medicina, en una misa en el Duomo. Al mirar

* Magíster en Filosofía (Epistemología y Filosofía de la Ciencia) de la Universidad de Caldas (2016). Ingeniero de Materiales de la Universidad de Antioquia (2007). Docente de cátedra de la Institución Universitaria Pascual Bravo.

Correo electrónico: areareiza@gmail.com

1 Algunos autores distinguen entre experimentos imaginarios y de pensamiento. El primero, aquellos que plantean paradojas, y así, el cambio de los aparatos conceptuales; y el segundo, como aquellos experimentos que lleva a supuestos por limitaciones de accesibilidad (la luna, el vacío, etc.) o dificultad de medición por imprecisión de instrumentos de la época (Cf. Respectivamente: Kuhn, "Función”; Álvarez y Marquina). 
hacia el techo de la catedral, observó que las lámparas de velas oscilaban. Notó que cada vez que iban oscilando las lámparas, iban disminuyendo su velocidad. Cuando la velocidad era más o menos constante, empezó a tomar el tiempo. Según la leyenda, él tomaba el tiempo de oscilación de dos posible maneras: (i) con el pulso de la muñeca o, (ii) con el tempo musical del coro que interpretaba las melodías en la liturgia; pues, porque no es de desconocer que el padre de Galileo, Vincenzo Galilei, era un gran músico y, posiblemente, Galileo aprendió dicho arte. Entonces, cuando Galileo tomaba el tiempo, se dio cuenta que el movimiento no dependía de la masa de las péndolas y tampoco de la amplitud; es decir, que a oscilaciones pequeñas, el periodo era constante. Ahora nosotros sabemos que esto es cierto, solo cuando el ángulo es aproximadamente igual al seno del ángulo $(\theta \approx \operatorname{sen} \theta)$. $O$ sea, que las amplitudes deben de ser muy pequeñas. Estas deben estar en el rango entre $0^{\circ}$ y $12^{\circ}$ para que se pueda dar la ley que se conoce como isocronismo, que puede resumirse como: el período de oscilación es independiente de la masa y de la amplitud depende, únicamente, de la longitud del hilo (Álvarez y Marquina).

Esta conclusión se extrae de la suposición que, una vez Galileo llega a casa, luego de la liturgia, comienza a experimentar con péndulos llevándolo a la ley de isocronía. Algunas razones que direccionan este experimento, tal como ha sido descrito, a pensar a que es mítico, son: (i) que estas lámparas, cuando Galilei estaba en la Universidad de Pisa, como estudiante de medicina, no existían. Estas lámparas las pusieron tres años después de la partida de Galileo de Pisa (Álvarez \& Marquina); y, (ii) la medición para calcular el tiempo para amplitudes tan pequeñas, requiere de un reloj de precisión. Por estas buenas razones, se puede suponer que dicho experimento no fue realizado, por lo menos, no de esta manera. Esto no pasó. Lo que uno se pregunta es: ćcómo ha quedado esto en el imaginario colectivo? Seguramente que por tradición. Con todo, y lo anterior, se puede identificar este experimento como mítico.

\section{Torre inclinada de Pisa}

Este es aún más reconocido: Según cuenta la tradición, Galileo, empleando la torre de Pisa, experimentó con un par de masas de hierro para intentar 
mostrar que los móviles caen al mismo tiempo. Y dice, además, que la torre era perfecta para la experiencia porque era inclinada. Ahora bien, ċcómo tomaba el tiempo? Se dice que de la misma forma que "calculó" el tiempo con las lámparas de la catedral: con el pulso de la muñeca o con los beats (b.p.m.) de la música que iba marcando mientras los objetos descendían. Ahora nos damos cuenta que el tiempo que tarda en caer un objeto desde la Torre de Pisa es 3,34 segundos. Pero surge la pregunta: ċcómo se puede medir 3,34 segundos con las formas sugeridas? No lo sabemos.

¿De dónde surge la referencia a este experimento? El historiador Alexandre Koyré señala que la única fuente bibliográfica que se dispone referente a esto es en el Racconto istorico de Vincenzo Viviani, y cita:

En esta época (1589-1590) se convenció de que la investigación
de los efectos de la naturaleza exige necesariamente un verdadero
conocimiento de la naturaleza del movimiento, conforme al axioma a
la vez filosófico y vulgar ignorato motu ignoratur natura; fue entonces
cuando, ante la gran indignación de todos los filósofos, demostró
-con la ayuda de experimentos, pruebas y razonamientos exactos- la
falsedad de numerosas conclusiones de Aristóteles sobre la naturaleza
del movimiento; conclusiones que hasta entonces eran tenidas por
claras e indudables. Así, entre otras, la de que las velocidades de los
móviles de la misma materia, pero de pesos diferentes y que se mueven
a través del mismo medio, no siguen en modo alguno la proporción
de su gravedad, tal como dice Aristóteles, sino que se mueven todos
con la misma velocidad. Lo que demostró por repetidos experimentos
hechos desde lo alto del campanario de Pisa en presencia de todos los
demás profesores y filósofos y de toda la Universidad.

[Demostró también] que las velocidades de un mismo móvil que cae a través de diferentes medios no siguen tampoco la proporción inversa de la densidad de estos medios, infiriendo esto a partir de consecuencias manifiestamente absurdas y contrarias a la experiencia sensible ("Galileo y el experimento" 200).

A partir del anterior fragmento han surgido cualquier cantidad de versiones, de las que cabe señalar el relato de Emile Namer por su altísimo drama, como nos lo señala Koyré: "Con increíble osadía, Galileo enviaba a Aristóteles a los polvorientos estantes de las bibliotecas. Proponía abrir el gran libro de la naturaleza y leer sus leyes con mirada fresca" ("Galileo y el experimento" 198). 
Continúa Namer:

Cuando Galileo supo que todos los otros profesores expresaban dudas referentes a las conclusiones del insolente innovador, aceptó el reto. Solemnemente invitó a estos graves doctores y a todo el cuerpo de estudiantes, en otros términos, a la Universidad entera, a asistir a uno de sus experimentos. Pero no en su marco habitual. No, éste no era suficientemente grande para él. Fuera, bajo el cielo abierto, en la ancha plaza de la catedral. Y la cátedra indicada claramente para estos experimentos era el Campanile, la famosa torre inclinada.

Los profesores de Pisa, como los de otras ciudades, habían sostenido siempre, conforme a la enseñanza de Aristóteles, que la velocidad de la caída de un objeto dado era proporcional a su peso.

Por ejemplo, una bola de hierro que pese cien libras y otra que pese una, lanzadas en el mismo momento, desde una misma altura, deben evidentemente tocar tierra en momentos diferentes y con toda seguridad la que pesa cien libras tocará tierra primero, puesto que justamente es más pesada que la otra.

Galileo, al contrario, pretendía que el peso no tenía nada que ver y que las dos tocarían tierra en el mismo momento.

Escuchar semejantes aserciones hechas en el corazón de una ciudad tan vieja y tan sabia era intolerable; y se pensó que era necesario y urgente afrentar públicamente a este joven profesor que tenía una opinión tan elevada de sí mismo y darle una lección de modestia de la que se acordase hasta el final de su vida.

Doctores con los largos trajes de terciopelo y magistrados que parecían querer ir a una especie de feria de pueblo abandonaron sus diversas ocupaciones y se mezclaron con los representantes de la Facultad dispuestos a burlarse del espectáculo fuera cual fuera el final.

Lo más extraño quizás de toda esta historia es que no se le ocurrió a nadie hacer el experimento por sí mismo antes de llegar a la plaza. Atreverse a poner en duda algo que Aristóteles había dicho, era nada menos que una herejía a los ojos de los estudiantes de esta época. Era un insulto a sus maestros y a ellos mismos, una desgracia que podría excluirlos de las filas de la élite. Es indispensable tener presente constantemente esta actitud para apreciar claramente el genio de Galileo, su libertad de pensamiento y su valor, y estimar en su justo mérito el sueño profundo del que la conciencia humana debía despertarse. iQué esfuerzo, qué luchas eran necesarias para dar nacimiento a una ciencia exacta!

Galileo subió las escaleras de la torre inclinada, con calma y tranquilidad a pesar de las risas y gritos de la multitud. Comprendía bien la importancia del momento. En lo alto de la torre, formuló una vez más el problema con toda exactitud. Si los cuerpos al caer llegaban a tierra al mismo tiempo, había conseguido la victoria, pero si llegaban en 
momentos diferentes, serían sus adversarios quienes tendrían razón. Todo el mundo aceptó los términos del debate. Gritaban: "Haced la prueba". Había llegado el momento. Galileo lanzó las dos bolas de hierro. Todos los ojos miraban arriba.

Un silencio. Y se vio salir juntas las dos bolas, caer juntas y juntas tocar tierra junto a la torre (ctd en Koyré, "Galileo y el experimento" 198-199).

Con lo anteriormente descrito, se puede identificar que el experimento es un mito. De haber sido tan importante, como se ha señalado en los fragmentos anteriores, es de suponer que en algún fragmento de la misma obra de Galileo o en alguna carta se describiera el suceso. Pero no hay ningún otro registro del supuesto evento. Además, para la época ya se identificaba que la supuesta variación de la caída de los graves, se daría en móviles de densidad específicas diferentes, y la descripción que nos da Viviani de la experiencia, es con dos móviles del mismo elemento, hierro. Sin embargo, el experimento sí se realizó, y no fue Galileo, sino el jesuita Riccioli, y no en la torre de Pisa, sino en la torre Asinelli de Bolonia.

Luego de la muerte de Galileo, muchos se interesaron en su obra y se despertó una cierta inquietud por reproducir las experiencias de Galileo. Es así, como Riccioli -dos años después de la muerte de Galileo-, realiza una experiencia de caída de los graves desde la torre Asinelli, confirmando las teorías expuestas por Galileo (cf. Villatoro).

\section{Experimentos imaginarios}

Un experimento mental o imaginario es aquel que el pensador plantea con el propósito de establecer con claridad el fenómeno que pretende aclarar y, a su vez, sugerir aquellas paradojas que llevan a un cambio en el aparato conceptual que describía con anterioridad dicho fenómeno. Así, "el experimento imaginario es una de las herramientas analíticas esenciales que se emplean durante la crisis [en el conocimiento científico] y que contribuye a promover la reforma conceptual básica" (Kuhn, "Función” 287).

En la obra Diálogo sobre los dos sistemas máximos del mundo, Galileo, en la voz de Salviati, plantea a sus interlocutores, Simplicio y Sagredo, 
una paradoja. Esta paradoja es más o menos como sigue. Suponga dos planos, $\mathrm{AB}$ vertical y $\mathrm{AC}$ inclinado (como muestra la figura siguiente). $\mathrm{A}$ lo largo de estos planos se mueven dos objetos de igual masa, uno en caída libre y el otro en traslación pura sobre el plano inclinado. Salviati, le pide a sus interlocutores que, cuando los cuerpos llegan a los puntos $\mathrm{B}$ y $\mathrm{C}$, respectivamente, estos habrán adquirido el mismo ímpetu o velocidad necesaria para volver al punto desde el cual se dejaron caer -velocidad inicial igual a cero-; a lo que seguidamente les pregunta cuál de los dos cuerpos se mueve más rápido. Para mostrar la paradoja en la que se encuentran Sagredo y Simplicio, vamos a intentar comprender con claridad en qué consiste, con algunas modificaciones, no sustanciales, tanto en la descripción como en el planteamiento.

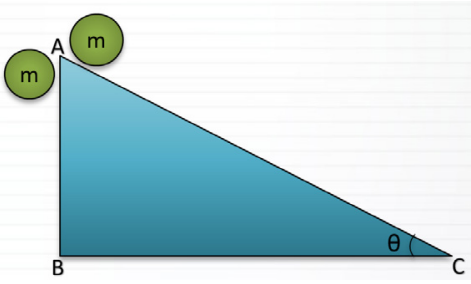

Ilustración de la caída de ambos móviles. Uno en caída y el otro sobre el plano inclinado (elaboración propia).

Para esto, y siendo anacrónicos, partiremos de lo que conocemos de la física, específicamente de la cinemática y de las leyes de movimiento, y calcularemos la velocidad final de los móviles en los puntos B y C, respectivamente:

Para el cuerpo que cae sobre la vertical:

$$
y_{f}=y_{i}+v_{i y} t+\frac{1}{2} a t^{2}
$$


Considerando las condiciones iniciales, la ecuación anterior queda de la forma:

$$
-h=-\frac{1}{2} g t^{2}
$$

Despejando, se obtiene el tiempo que tarda en descender hasta el punto B:

$$
t=\sqrt{\frac{2 h}{g}}
$$

Y, finalmente, reemplazando en la ecuación: , se obtiene la velocidad en el punto B. Esto es:

$$
v_{B}=-g \sqrt{\frac{2 h}{g}}
$$

Ingresando $g$ en la raíz, simplificando y tomando la magnitud de dicha velocidad, tenemos que:

$$
\left|v_{B}\right|=\sqrt{2 g h}
$$

Por otra parte, para el cuerpo sobre el plano inclinado, y aplicando la segunda Ley de Newton, se obtiene:

$$
\sum F_{x}: m g \operatorname{sen} \theta=m a
$$

Así, se puede hallar la aceleración del móvil, entonces: $a=g \operatorname{sen} \theta$.

Determinando la longitud del plano inclinado $(z)$ en términos de $h$, se tiene que:

$$
\operatorname{sen} \theta=\frac{h}{z} \rightarrow z=\frac{h}{\operatorname{sen} \theta}
$$


Tomando los cálculos anteriores y con una de las ecuaciones cinemáticas, se puede hallar la velocidad con la que el móvil llega a la base del plano inclinado, esto es:

$$
\begin{gathered}
v_{f}^{2}=v_{i}^{2}+2 a\left(x_{f}-x_{i}\right) \\
v_{f}^{2}=\frac{2 h g \operatorname{sen} \theta}{\operatorname{sen} \theta} \rightarrow \quad v_{f}=\sqrt{2 g h}
\end{gathered}
$$

Con los cálculos anteriores, se puede observar que las velocidades finales de ambos móviles son iguales, como inicialmente había sugerido Galileo a sus interlocutores en términos de ímpetu.

Ahora bien, retomando la pregunta que sugiere Galileo: ¿̇cuál de los dos cuerpos se mueve más rápido? Primero que nada, debemos precisar a lo que la pregunta se refiere, a lo que podemos decir: será más rápido aquel que abarca la misma distancia en menor tiempo.

Al tomar ambos movimientos, se debe considerar un referente de distancia que ayude a tener una relación comparativa entre ellos. Para esto, se tomará la distancia $h$ como longitud estándar o patrón. Así: (i) al dejar caer ambos cuerpos desde A, el móvil que se desplaza sobre la vertical, tardará menos tiempo $\left(t=\sqrt{\frac{2 h}{g}}\right)$ en recorrer la misma distancia $h$ que el móvil que se mueve sobre el plano $\left(t=\frac{1}{\operatorname{sen} \theta}\left(\frac{\sqrt{2 h}}{\theta}, 0<\theta<\frac{\pi}{2}\right)\right.$, ya que la distancia $\mathrm{AB}$ es menor que $\mathrm{AC}$; sin embargo, (ii) si se toma la misma longitud estándar o patrón $(h)$ desde el vértice $\mathrm{C}$ hacia $\mathrm{A}$, el móvil de la perpendicular tardará mayor tiempo que el móvil que se mueve por plano inclinado. Finalmente, y como otra posibilidad, (iii) si en algún punto del segmento AC se mide la distancia patrón $(h)$ en puntos intermedios del plano inclinado, entonces serán iguales los tiempos necesarios para que ambos móviles recorran la misma distancia. De este modo, la posible respuesta a la pregunta ha llevado a tres situaciones diferentes quedando planteada la paradoja.

Este tipo de paradojas son las que nos plantea Galileo Galilei como punto de partida para el nacimiento de una nueva ciencia. Entonces, 
de este modo, se puede afirmar que este tipo de experimentos se dan como una conversación del pensador con las posibilidades -anomalías-, redireccionando a un cambio en el aparato conceptual. Con el surgimiento de varias anomalías que evidencian dificultades con el paradigma vigente, un experimento mental es, quizás, el mejor experimento para salir de ellas, sabiendo que después de una crisis vendrá una revolución en la ciencia y en el pensamiento (cf. Kuhn, Estructura).

\section{Experimentos reales}

\section{Plano inclinado y proyecciones}

Respecto a los experimentos reales, ¿cómo determinar que son reales? Desde la obra misma de Galileo es difícil identificarlo. Algunos historiadores, como Stillman Drake, Peter Dear, James MacLachland, entre otros, han tomado algunos folios publicados en la Biblioteca Nacional Central de Florencia a partir de los manuscritos de Galileo, tanto de sus notas como de sus cartas. Un folio muy tratado entre los historiadores y filósofos de la ciencia es el folio $116 \mathrm{v}$, el cual permite identificar la posibilidad de la experimentación en Galileo.

[ Cálculos realizados por Galilei (en notación moderna)]

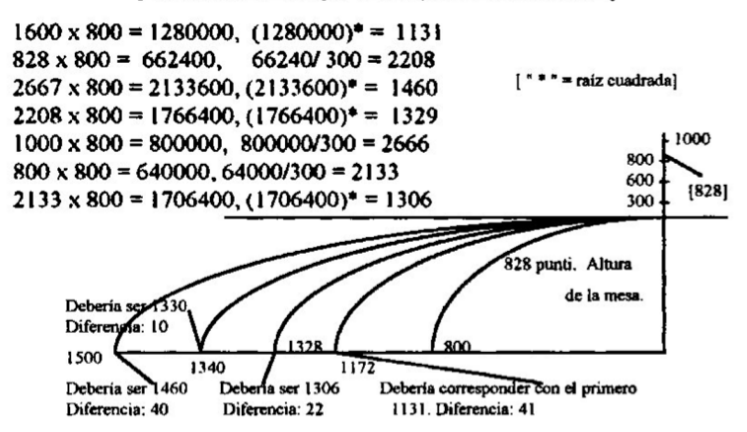

Ilustración del folio 116v. Galileo confronta los valores de las proyecciones horizontales con un anterior análisis matemático (Álvarez y Posadas 70) 
El experimento, que se encuentra descrito en los Discorsi (Galilei, "Diálogo sobre dos nuevas ciencias"), tiene unas especificaciones técnicas muy complejas. Como puede verse en la siguiente descripción:

En un tablón, o si se quiere, en una viga de madera de unas 12 brazas de largo y media braza de ancho por un lado y 3 dedos por el otro, se había tallado, en esta anchura menor, un canalito poco más ancho de un dedo; habiéndolo trazado muy recto, y, después de pegarle en su interior un pergamino bruñido y lustrado todo lo posible para tenerlo muy pulido y liso, se hacía descender por él una bola de bronce durísimo, muy redonda y pulida; después de inclinar la viga, elevando sobre el plano horizontal uno de sus extremos una o dos brazas a voluntad, se dejaba [...] descender por dicho canal la bola, anotando, en la manera que indicaré enseguida, el tiempo que empleaba para recorrerlo todo, en el cual jamás se encontraba una diferencia ni siquiera de la décima parte de una pulsación [...]. En lo que respecta a la medida del tiempo, se empleaba un gran cubo lleno de agua, suspendido [en] lo alto, del cual, por un delgado canalito soldado en su fondo, caía un fino hilo de agua que se recogía en un pequeño vaso, durante todo el tiempo en que la bola descendía por el canal y por sus partes. Luego, las partículas de agua recogidas de este modo, se iban pesando cada vez con una balanza exactísima, dándonos las diferencias y proporciones de sus pesos, la diferencia y proporciones de los tiempos; y esto con tal precisión que, como ya he dicho, repetidas una y otra vez estas operaciones, nunca diferían de modo apreciable (ctd en Romo 16).

Comparando la descripción anterior y el manuscrito, Galileo espera mantener la proporción entre la altura y el cuadrado de la distancia, entonces saca una relación a partir de los datos que obtiene, tomando como referencia la proporcionalidad de los valores mínimos. En la vertical se puede observar un valor de 300 punti y en la horizontal un valor de 800 punti como valores mínimos (ver imagen anterior). Él dejaba caer los móviles sobre un plano inclinado e iba tomando los datos. En el folio, Galileo señala valores obtenidos y agrega: "deberían" -como se observa en los apuntes del folio- de ser otros. Esto plantea, que este experimento ya lo tiene referenciado a partir de un análisis matemático previo.

Anteriormente esto no se daba, principalmente porque el aristotelismo, doctrina de pensamiento instaurado, solía señalar que cuando hablábamos de las cosas, se hablaba del modo tal cual como ellas se 
presentaban. Galileo, en contraste, comienza a crear artefactos para poder confrontar sus supuestos. Y, a partir de sus ideas, obtenía expresiones que contrastaban entre la experiencia y los supuestos matematizados.

Esto es importante, porque la ciencia es una conversación constante que se da con la naturaleza. Como señala Koyré ("Galileo y Platón"): la naturaleza nos habla en un lenguaje, y ese lenguaje es matemático -geométrico-. Ya Galileo hablaba un lenguaje matemático.

Por otra parte, Koyré ("De motu gravium") señala que este experimento es imposible porque lo que describe por medio de Salviati, no es viable de obtener con tal rigor, ya que el nivel de exactitud en las mediciones es demasiado, sumando que, lograr dichas superficies perfectas es imposible. No olvidemos que el desarrollo innovador en el telescopio de Galileo fue el material y el pulido de los lentes que empleaba; adicional, el trabajo artesanal de la época era brillante, lo que nos hace pensar que la descripción del experimento, es, por lo menos, posible.

\begin{tabular}{cccc}
\hline $\begin{array}{c}\text { Altura del } \\
\text { plano }(\mathrm{H})\end{array}$ & $\begin{array}{c}\text { Proyección horizontal } \\
\text { experimental }\left(\mathrm{D}_{E}\right)\end{array}$ & $\begin{array}{c}\text { Proyección horizontal } \\
\text { usando la RMP }(\mathrm{D})\end{array}$ & $\begin{array}{c}\text { Error } \\
(\%)\end{array}$ \\
\hline 300 & 800 & - & - \\
600 & 1172 & 1131 & 3.6 \\
800 & 1328 & 1306 & 1.7 \\
828 & 1340 & 1329 & 0.8 \\
1000 & 1500 & 1460 & 2.7 \\
\hline Los datos están en punti. El error promedio es de $2.20 \%$.
\end{tabular}

Error entre los datos obtenidos por Galileo reportados en el manuscrito y los esperados por el análisis matemático (Álvarez y Posadas 71)

Analizando la relación de los datos obtenidos por Galileo en el folio (como puede verse en la tabla anterior), se puede observar el margen de error entre los datos obtenidos por la experiencia y los esperados por la relación matemática. El error es de 2,2\%, un error que permite concluir que dicha experiencia encarna el experimento moderno por las siguientes razones: (i) alta coincidencia numérica entre los datos de Galileo y las reproducciones contemporáneas; (ii) resaltar con mayor precisión las variables obtenidas 
como de aquellas que concurrieron en el experimento, y, (iii) la confrontación de una hipótesis con valores empíricos (cf. Álvarez y Posadas).

Como se ha señalado, Stillman Drake toma la descripción de Galileo, en la voz de Salviati, y hace la experiencia, tomando la misma expresión y la misma discusión como lo describe Galileo en los Discorsi. Él obtiene valores similares y se da cuenta que es posible; de hecho, lo contrasta con la misma expresión que obtiene Galileo (ver los resultados obtenidos en la tabla siguiente), pero apelando a recursos modernos, por ejemplo, el del tiempo. El tiempo para Drake es medido con los dispositivos actuales y de alta precisión.

\begin{tabular}{|c|c|c|c|c|c|}
\hline I & II & III & IV & V & VI \\
\hline $\begin{array}{l}\text { Altura } \\
\text { del } \\
\text { plano } \\
\text { (H) }\end{array}$ & $\begin{array}{c}\text { Velocidad } \\
\text { terminal }\end{array}$ & $\begin{array}{c}\text { Proyección } \\
\text { horizontal } \\
\text { teórica } \\
\left(\mathrm{D}_{T}\right)\end{array}$ & $\begin{array}{c}\text { Proyección } \\
\text { horizontal } \\
\text { exp. } \\
\left(\mathrm{D}_{E}\right) \\
\end{array}$ & $\begin{array}{l}\text { Proyección } \\
\text { horizontal en } \\
\text { elf. } 116 \mathrm{v} \\
\text { (D) }\end{array}$ & $\begin{array}{c}\text { Error } \\
\text { entre } \\
\text { IV y V } \\
(\%)\end{array}$ \\
\hline 300 & 2022 & 805 & 808.8 & 800 & -0.7 \\
\hline 600 & 2860 & 1139 & 1144.0 & 1172 & +2.8 \\
\hline 800 & 3303 & 1315 & 1321.2 & 1328 & +1.0 \\
\hline 828 & 3360 & 1338 & 1344.0 & 1340 & +0.2 \\
\hline 1000 & 3692 & 1470 & 1476.8 & 1500 & +2.0 \\
\hline
\end{tabular}

Datos obtenidos de la reproducción experimental de Drake del folio 116v (Álvarez y Posadas 72).

Con todo, hasta ahora, podemos volver al planteamiento inicial sobre lo que es un experimento. José Romo, al respecto, señala lo que es un experimento para Peter Dear:

[...] un experimento es: "un suceso histórico en el que un investigador examina [experiences] la conducta de un dispositivo o aparato diseñado y utiliza, o podría utilizar, un informe de ese suceso histórico como elemento en la construcción de un argumento que pretende establecer o promover una aportación al conocimiento. Un experimento, por consiguiente, es solo un experimento si aparece como tal en el discurso científico, o podría serlo dado el contexto en el que se creó' (ctd. en Romo 7). 
Se pueden analizar dos frases de la afirmación de Dear: (i) es un suceso histórico -en la obra de Galileo no hay un suceso, pero en el manuscrito sí-, y, (ii) si el experimentador no describe su práctica como tal, esta no puede considerarse como experimento. Este es el caso de Galileo. Así, según la descripción que hace Dear sobre lo que es un experimento, no podría afirmarse que Galileo experimentara.

Ahora bien, tomando la descripción de la obra los Discorsi, mencionada anteriormente, la cual Dear conoce bien, este advierte: "En vez de describir un experimento específico [...] llevados a cabo en un momento concreto [...], Galileo dice simplemente que, utilizando un dispositivo determinado, descubrió que los resultados concordaban exactamente con sus supuestos teóricos [...]" (ctd. en Romo 7). Con esto, la experiencia llevada a cabo por Galileo, según Dear, no dista de la experiencia del aristotelismo.

Con todo, y esto, chacía Galileo experimentos?, Dear señala:

Cuando Galileo hacía descender esferas por planos inclinados y las lanzaba desde los bordes de las mesas para medir las distancias que recorrían, no estaba haciendo "experimentos" en el sentido moderno, a menos que hiciera estas cosas para apuntalar su presentación formal en sus escritos como sucesos históricos discretos. Y este no es el caso (ctd. en Romo 8; énfasis de Dear).

Entonces, para Dear, se entenderá como "experimento" aquellas descripciones explícitas tanto de los dispositivos empleados como, también, aquellos resultados numéricos concretos. En este sentido, el manuscrito denominado folio $116 \mathrm{v}$, cumpliría con la descripción dada por Dear, excepto que no está publicada en la obras de Galileo. Adicional, como señala Romo:

[...] cuando se habla de "experimentos en Galileo", o bien se comete un anacronismo, en la medida en que se proyecta sobre su práctica una noción de experimento construida posteriormente, o bien se hace suponiendo implícitamente que la obra publicada contiene elementos retóricos que los manuscritos [...] permitirán determinar, independientemente de lo que decidiera publicar (9; énfasis de Romo). 
En general, si consideramos el folio $116 \mathrm{v}$ y lo descrito por Galileo en los Discorsi, se podría suponer que los resultados no podrían ser expuestos de forma directa, ya que, como se puede señalar en dicho folio, los resultados no eran los esperados según la relación entre la distancia al cuadrado $\left(\mathrm{D}^{2}\right)$ con la altura $(\mathrm{H})$ y los valores de proporcionalidad de los valores mínimos de medición. Lo que se puede afirmar es que Galileo, en el folio, lo que intenta, según Drake, es contrastar una noción de persistencia del movimiento horizontal que se ve obligado a rechazar según los datos obtenidos; sin embargo, para otros, el experimento del plano inclinado busca precisar la ley de la caída de los graves, probando una trayectoria parabólica.

Para lo que aquí queremos señalar, la experiencia realizada por Galileo, nos permite afirmar que la acción llevada a cabo en el folio, demuestra que si quizá no se puede contemplar dicha acción en lo que se entiende como un experimento moderno, sí es muy próximo a lo que esto es (cf. Álvarez y Posadas).

\section{El vino y el agua}
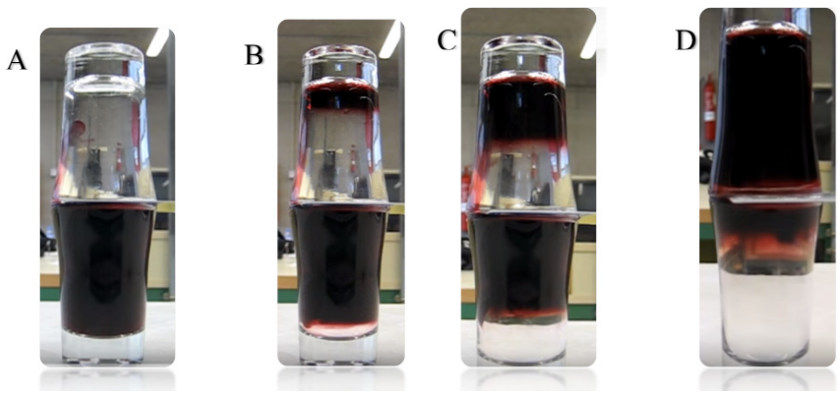

Experiencia realizada durante la charla (Paredes) ${ }^{2}$.

2 Imagen reconstruida y recuperada de Paredes. 
Finalmente, una experiencia bastante controvertida en la literatura especializada, es la del vino y el agua. La descripción de la experiencia es como sigue:

Si lleno de agua un globo de cristal en el que se ha hecho un agujero tan pequeño como el grosor de una paja, y si, una vez lleno, lo vuelvo con el agujero hacia abajo, el agua, a pesar de su peso y prontitud para descender en el aire, y el aire, igualmente dispuesto a elevarse en el agua dada su extrema ligereza, no se ponen de acuerdo, la una por caer, saliendo por el orificio y el otro para subir, entrando por el mismo. Por el contrario, permanecen, más bien, los dos hostiles y desconfiados. Si presento, por el contrario, a aquel orificio un vaso de vino tinto, que es en una medida casi insensible más ligero que el agua, lo veremos inmediatamente elevarse lentamente a través del agua en forma de trazos rojizos, mientras que el agua, con la misma lentitud, descenderá por el vino, por lo que el agua caerá toda al fondo del vaso colocado debajo. Por todo ello, ćqué otra cosa decir, que se pueda concluir de lo dicho, sino que hay una incompatibilidad entre el agua y el aire, misteriosa para mí, aunque tal vez...? (ctd. en Naranjo 21).

En el artículo "El 'De motu gravium' de Galileo: Del experimento imaginario y de su abuso", Koyré, en el apéndice dedicado a examinar sobre los abusos del experimento imaginario, señala respecto a esta experiencia: (i) el experimento es imposible, (ii) para ser posible, sin que haya mezcla, deberían ser inmiscibles, (iii) se requieren dos orificios, y no uno, (iv) provistos con capilares orientados, ( $v$ ) habrá mezcla en cualquier caso, (vi) Galileo no mezcló vino y agua, el vino era para él "la encarnación de la luz del sol", (vii) por rumores, reconstruye el experimento imaginariamente, (viii) admitiendo como algo indudable la incompatibilidad de agua y vino, (ix) incompatibilidad "esencial y total", $(x)$ los vinos del siglo XVII eran inmiscibles en agua, (xi) al menos los vinos tintos, (xii) como lo es el aceite (cf. Naranjo).

Ahora, aquí, en esta conferencia, podremos entrar en la discusión y, con la experiencia aquí realizada, afirmar que el experimento es totalmente posible y real. 
Ahora, a modo de explicación, ¿̇por qué se da esto? Cuando consideramos la configuración aquí mostrada (ver imagen): agua en el recipiente inferior, vino en el superior y una pequeña lámina que separa ambas sustancias dejándolas interactuar por un pequeño orificio en el extremo de ambos recipientes, se puede observar, como lo describe Galileo, una línea rojiza que comienza el ascenso hacía el agua mientras el agua desciende por un camino de deslizamiento entre las dos sustancias. Esto se conoce como flujo laminar y consiste en el paso de ambos fluidos que se desplazan como láminas, ascendiendo la menos densa y descendiendo la más densa. En este caso, como puede observarse, el vino asciende y el agua desciende.

Al analizar el texto de Koyré, y considerando la obra del autor ruso, podemos identificar así tres premisas centrales, como señala Naranjo: (i) la realidad imprecisa, (ii) la experiencia inútil, y, finalmente, (iii) el carácter abstracto de las leyes físicas. Para Koyré, lo que nosotros conocemos del estado de cosas, son solo las teorías establecidas. Toda experiencia que sea antes de la ley no importa, lo que importa es la ley. Por estas razones, la experiencia del vino y el agua, aquí presenciado, lo relaciona Koyré como un experimento imaginario.

Como hemos podido mostrar a lo largo de la conferencia, y como afirma Drake, Galileo Galilei no es precisamente el padre de la experimentación, pero si es la certificación del uso de experiencias para la contrastación de sus conjeturas e hipótesis matemáticas o, mejor, el precursor de la experiencia -experimentación- moderna: concibiendo cuerpos reales por cuerpos ideales; el espacio real por el espacio geométrico; el uso de las matemáticas -geometrización- para la valoración y verificación de sus supuestos teóricos; el empleo, diseño y construcción de artefactos para la validación de sus supuestos - como puede apreciarse en su obra y en los folios-, entre otros aspectos.

Es así, como a partir de los aportes de estos grandes pensadores la humanidad se asienta, y sobre hombros de gigantes, levanta el vuelo. 


\section{Lista de referencias}

Álvarez, José Luis, y Yolanda Posadas. "La obra de Galileo y la conformación del experimento en la física". Revista mexicana de física 49.1 (2003): 62-74.

Álvarez, José Luis, y José Ernesto Marquina. "Los experimentos de Galileo”. Ciencias. Revista de la cultura científica 26 (1992): 1526.

Ferrater Mora, José. Diccionario de Filosofía. Barcelona: Ariel, 2004.

Galilei, Galilei. "Diálogo sobre dos nuevas ciencias”. Ed. Stephen Hawking. A hombros de gigantes. Barcelona: Crítica, 2015. 357-553 . Diálogo sobre los dos máximos sistemas del mundo ptolemaico y copernicano. Madrid: Alianza, 1995.

Koyré, Alexandre. "El 'De motu gravium' de Galileo: Del experimento imaginario y de su abuso". Estudios de historia del pensamiento científico. México: Siglo XXI, 2007. 206-57.

. "Galileo y el experimento de Pisa: a propósito de una leyenda". Estudios de historia del pensamiento científico (págs. 196-206). México: Siglo XXI, 2007. 196-205.

. "Galileo y Platón". Estudios de historia del pensamiento científico. México: Siglo XXI, 2007. 150-179.

Kuhn, Thomas S. La estructura de las revoluciones científicas. México: Fondo de Cultura Económica, 2006.

. "La función de los experimentos imaginarios". La tensión esencial. México: Fondo de Cultura Económica, 1982. 263-89.

- Segundos pensamientos sobre paradigmas. Madrid: Tecnos, 1978.

Naranjo, Jorge Alberto. Los trabajos experimentales de Galileo Galilei. Bogotá: Universidad Nacional de Colombia, 1988.

Paredes, Sergio. "Movido no agitado". Cluster de divulgación científica, 10 may. 2009. <http://cluster-divulgacioncientifica.blogspot.com. co/2009/05/movido-no-agitado.html $>$.

Romo, José. “¿Hacía Galileo experimentos?” Theoria 52 (2005): 5-23.

Villatoro, Francisco R. "El experimento de Galileo en la torre de Pisa lo realizó Riccioli en la torre Asinelli de Bolonia". La Ciencia de la Mula Francis, 26 ago. 2012. <http://francis.naukas.com/2012/08/26/ 
nota-dominical-el-experimento-de-galileo-en-la-torre-de-pisa-lorealizo-riccioli-en-la-torre-asinelli-de-bolonia/> .

"Working Level of Folio 116v". Museo Galileo (Biblioteca Nazionale Centrale, Florence Istituto e Museo di Storia della Scienza). Florencia. < http:/www.imss.fi.it/ms72/HTML/F116_V/M116_V. HTM > . 